\title{
2015 TMS Journal Impact Factors; TMS Welcomes New Members
}

\section{new and noteworthy at TMS}

Turn to this regular JOM feature for information on new TMS initiatives, updates and overviews of TMS activities, and news from the field that impacts TMS and its members. To submit news items for consideration, contact Lynne Robinson, JOM Contributing Editor, at Irobinson @tms.org.

\section{Impact Factors Released for TMS Journals}

The steady, upward trend in Impact Factor (IF) scores continued for TMS's long-established journals with the June
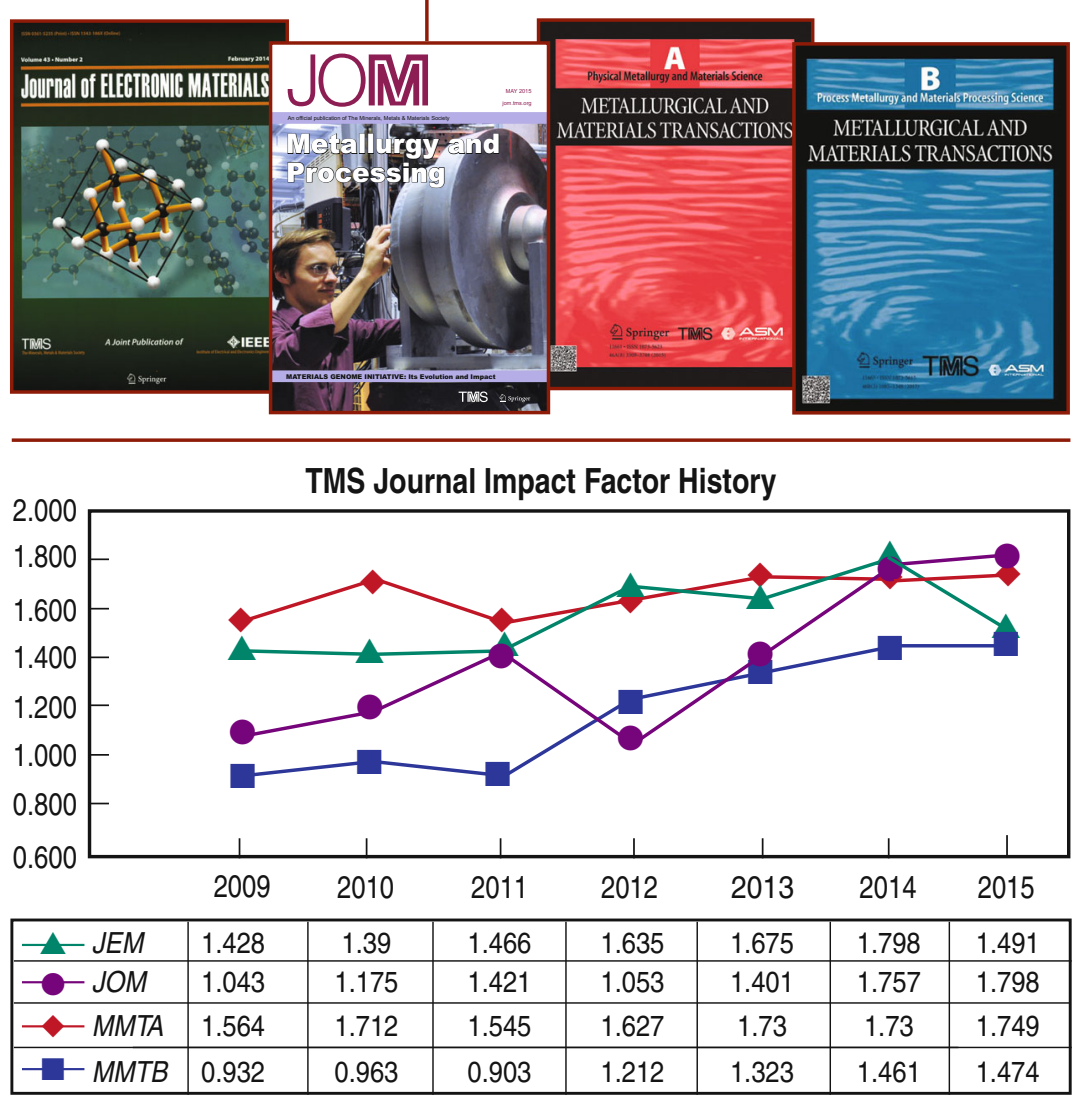

Figure 1. TMS journals have demonstrated an ongoing increase in Impact Factor scores over time.

Table I: Growth in TMS Journal Article Citations

\begin{tabular}{|c|c|c|c|}
\hline Journal & Total Citations 2013 & Total Citations 2014 & Total Citations 2015 \\
\hline JEM & 6,636 & 7,438 & 7,536 \\
\hline JOM & 3,263 & 3,894 & 4,411 \\
\hline MMTA & 17,131 & 19,262 & 20,111 \\
\hline MMTB & 4,074 & 4,620 & 4,837 \\
\hline
\end{tabular}

release of the 2015 IF numbers by the Thomson Reuters ISI Web of Knowledge citation measurement index. Impact Factors are based on the number of citations of a journal's materials divided by the number of citable materials published by that journal.

Metallurgical and Materials Transactions $A$ and $B$, as well as $J O M$, notched an increase, while the Journal of Electronic Materials experienced a decrease. What follows are the $2015 \mathrm{IF}$ numbers for TMS journals, with the 2014 IF in parentheses:

- JOM: 1.798 (1.757)

- Metallurgical and Materials Transactions A: 1.749 (1.73)

- Metallurgical and Materials Transactions B: 1.474 (1.461)

- Journal of Electronic Materials: 1.491 (1.798)

While IF can vary from year to year for a variety of reasons, TMS journals have generally tracked in a positive direction (Figure 1), along with corresponding growth in total citations (Table I). TMS journals also achieved strong placement in ISI Web of Knowledge ranking by subject categories (Table II), as determined by their Impact Factors. Authors often refer to the IF and subject ranking of a journal for guidance when seeking to maximize exposure of their work.

"TMS journals are fundamental to the Society's service and value to its members and the professional community as a whole," said James J. Robinson, TMS Executive Director. "Impact Factor is one of many indicators of the growing influence of each of these journals as a showcase for some of the finest work in our field. It is also yet another validation of the hard 
work and dedication of the editors, peer reviewers, authors and others actively engaged in the current and future success of these important periodicals."

To access TMS's entire library of journals, visit the TMS Knowledge Resource Center at knowledge.tms.org. By logging in to SpringerLink via the TMS website, TMS members can read the current issues and archives of all of these journals for free.
Table II: TMS Journal Rank by Subject Category

\begin{tabular}{|c|c|c|c|c|c|}
\hline Catagory Name & $\begin{array}{l}\text { Total Journals } \\
2015\end{array}$ & JOM & MMTA & MMTB & JEM \\
\hline Materials Science, Multidisciplinary & 271 & $\overline{116}$ & 126 & 152 & 151 \\
\hline Metallurgy \& Metallurgical Engineering & 73 & 13 & 14 & 20 & - \\
\hline Mineralogy & 29 & 11 & - & - & - \\
\hline Mining \& Mineral Processing & 21 & 5 & - & - & - \\
\hline Engineering, Electronical \& Electronic & 255 & - & - & - & 108 \\
\hline Physics, Applied & 145 & - & - & - & 80 \\
\hline
\end{tabular}

\section{TMS Welcomes New Members}

The TMS Board of Directors approved professional membership for 668 new members at its May 2016 meeting. Due to the size of this new member list, JOM published the first half in its August 2016 issue. The rest of our new members approved in May are presented below. Please join us in congratulating and welcoming them to all of the privileges and benefits of TMS membership.

Ma, Dong; Oak Ridge National Laboratory, United States

Maalekian, Mehran; Aim Metals \& Alloys, Canada

Mabbett, lan; Swansea University, United Kingdom

Magarotto, Giovanni; T.T. Tomorrow Technology, Italy

Maguire, Michael; Sandia National Laboratory, United States

Maniruzzaman, Mohammed; Caterpillar Incorporated, United States

Manzoni, Anna; Helmholtz Zentrum Berlin, Germany

Martinez, Saez Enrique; Los Alamos National Laboratory, United States

Maruoka, Nobuhiro; Tohoku University, Japan

Marx, Michael; Saarland University, Germany

Matsunaka, Daisuke; Shinshu University, Japan

McClelland, Zackery; United States Army Engineer Research and Development Center, United States

McEnerney, Bryan W.; Jet Propulsion Laboratory, United States

McHugh, Peter; National University of Ireland Galway, Ireland

McIntosh, Grant; Light Metals Research Centre/University of Auckland, New Zealand
McKerns, Michael M.; California Institute of Technology, United States

Mehrabi, Hamid; Cranfield University, United Kingdom

Meidani, Hossein; Switzerland Global Enterprise, Switzerland

Meiring, Hans; Southwire Company, United States

Melling, Erling; Hycast AS, Norway

Mendelev, Mikhail; Ames Laboratory, United States

Meng, Wen Jin; Louisiana State University, United States

Meydanoglu, Onur; Assan Aluminyum Sanayi ve Ticaret A.S., Turkey

Minnich, Austin; California Institute of Technology, United States

Minter, Jonathan; Indium Corporation, United States

Mishra, Raja K.; General Motors Research and Development Center, United States

Miyai, Seiichi; Japan Science and Technology Agency, Japan

Moon, Byung; Korea Institute of Industrial Technology, South Korea

Moreno, Marc; Institut Jean Lamour/ CNRS, France

Moss, Tyler; Bechtel Marine Propulsion Corporation, United States

Mota, Fatima; Institut Materiaux Microelectronique Nanosciences de Provence, France
Moulin, Jean-Michel; ArcelorMittal, Luxembourg

Mousseau, Normand; Universite de Montreal, Canada

Mulugeta, Yigezu; Addis Ababa University, Ethiopia

Nai, Mui Ling Sharon; Singapore Institute of Manufacturing Technology, Singapore

Najmaei, Sina; U.S. Army Research Laboratory, United States

Nam, Chang-Yong; Brookhaven National Laboratory, United States

Namilae, Sirish; Embry Riddle Aeronautical University, United States

Nash, Robert A.; Gillespie \& Powers Incorporated, United States

Neises, Martina U.; German Aerospace Center, Germany

Nguyen-Thi, Henri; Aix-Marseille University, France

Nichols, Ashley; 3D Material Technologies, United States

Niedworok, Carsten; IBAU Hamburg, Germany

Noethen, Nicole; EKW GmbH, Germany

Okubo, Satoru; Japan Oil, Gas and Metals National Corporation, Japan

Olsson, Par; KTH Royal Institute of Technology, Sweden

Oluwatobi, Samuel; University of Johannesburg, South Africa 
Omotosho, Olugbenga; Covenant University, Ota, Nigeria

Orlikowski, Daniel; Lawrence Livermore National Laboratory, United States

Orozco, Alberto; University of Manchester, United Kingdom

Osten, Karel J.; GRD Minproc, Australia

Otero, Michael; Sohar Aluminium, Oman

Pacheco, Vicente; Fraunhofer IFAM, Germany

Paci, Matteo; Profilglass, Italy

Paden, William; Ascend Performance Materials, United States

Palukuri, Nageswara Rao; Meddy Institute of Technology and Management, Hyderabad, India

Pan, Xiaolin; Northeastern University, China

Pan, Zhengwei; University of Georgia, United States

Panat, Rahul; Washington State University, United States

Pandelaers, Lieven; KU Leuven, Belgium

Papandrew, Alexander; University of Tennessee, United States

Paquit, Vincent; Oak Ridge National Laboratory, United States

Park, Chanman; Naval Postgraduate School, United States

Park, Harold; Boston University, United States

Park, Hyunsik; Korean Institute of Geoscience and Mineral Resources, South Korea

Park, Jeong-Yong; Korean Institute of Geoscience and Mineral Resources, South Korea

Park, Se Young; LG Display, United States

Patel, Jayesh B.; University BCAST, United Kingdom

Patel, Maulik; University of Tennessee, United States

Pearen, Dale; Hatch Ltd, Canada

Perez, Prado; Maria Teresa; IMDEA Materials Institute, Spain
Perez Trujillo, Francisco J.; Universidad Complutense, Spain

Perez-Reche, Francisco; University of Aberdeen, United Kingdom

Peters, Baron; University of California, United States

Petersen, Stephan; Global Telecommunication, Germany

Petrov, Roumen; Ghent University, Belgium

Phan, Manh-Huong; University of South Florida, United States

Pirard, Eric; University of Liege, Belgium

Pisch, Alexander; LafargeHolcim Research and Development, France

Polle, Shane; Emirates Global Aluminium, United Arab Emirates

Pons, Albin; Quintus Technologies LLC, United States

Pourroy, Genevieve; Institut de Physique et Chimie des Matériaux de Strasbourg, France

Prater, Tracie J.; National Aeronautics and Space Administration, United States

Price, Jason; Hazen Research, Incorporated, United States

Qin, Zhengjun; Southern Cross Property Consultants, China

Rabbi, Fazle; University of South Carolina, United States

Rager, Matthew; Oak Ridge National Laboratory, United States

Rajamani, Raj K.; University of Utah; United States

Rak, Zsolt; North Carolina State University; United States

Raman, Sumathy; Exxon Mobil Research and Engineering, United States

Ramanathan, Shriram; Purdue University, United States

Ramirez Ortega, Pedro; Universidad Tecnologica De Tul, Mexico

Ramirez-Argaez, Marco A.; Universidad Nacional Autonoma de Mexico, Mexico

Rao, Ramesh C.; University of Alabama, United States

Ray, David; Smelter Service, United States

Ray, Dipanjan; Los Alamos National Laboratory, United States
Reiss, Rebecca; New Mexico Technology, United States

Restrepo, David; Purdue University, United States

Reynolds, Tony; University of South Carolina, United States

Ridwan, Muhammad; PT. Indonesia Asahan Aluminium, Indonesia

Rigopoulos, loannis; University of Cyprus, Republic of Cyprus

Robelin, Christian; Ecole Polytechnique de Montreal, Canada

Roberts, Nicholas; Utah State University, United States

Rock, Ben; United States Naval Research Laboratory, United States

Roesler, Gernot; Montanuniversitaet Leoben, Austria

Roine, Antti JK; Outotec, Finland

Rondinelli, James; Northwestern University, United States

Rosseel, Thomas; Oak Ridge National Laboratory, United States

Rozman, Kyle; National Energy Technology Laboratory, United States

Rudin, Sven; Los Alamos National Laboratory, United States

Ruiz, Jose; Mersen USA, United States

Runnels, Brandon; University of Colorado, United States

Ryan, Vivian; Northrop Grumman, United States

Rye, Ketil A.; Alcoa, Norway

Ryu, Ho Jin; Korea Advanced Institute of Science \& Technology, South Korea

Sa, Injin; Korea Atomic Energy Research Institute, South Korea

Saito, Yoshiyuki; IHI Corporation, Japan

Samuel, Johnson; Rensselaer Polytechnic Institute, United States

Sandfeld, Stefan; University of Erlangen-Nuremberg, Germany

Sansoucie, Michael; NASA Marshall space Flight Center, United States

Sassaman, Katie; United States

Sato, Nobuaki; Tohoku University, Japan 
Sato, Yuzuru; Tohoku University, Japan

Sauzay, Maxime; French Atomic Energy Commission, France

Saxena, Surendra K.; Florida International University, United States

Saxena, Vivek; Vedanta Limited, India

Schaffer, Jeremy; Fort Wayne Metals Research, United States

Scherrer, Hubert; University of Lorraine, France

Schirk, Peter Grant; BHS-Sonthofen Incorporated, United States

Schnabel, Volker; RWTH Aachen University, Germany

Schneider, Stephan; German Aerospace Center, DLR, Germany

Schniepp, Hannes; The College of William \& Mary, United States

Schwaiger, Ruth; Karlsruhe Institute of Technology (KIT), Germany

Scott, Russell; Honda R\&D Americas, Incorporated, United States

Scott, Garney B.; Scepter, United States

Seepersad, Carolyn; University of Texas at Austin, United States

Seko, Atsuto; Kyoto University, Japan

Shi, Qianying; University of Michigan, United States

Shi, Xun; Shanghai Institute of Ceramics, China

Shiotsuki, Katsuhiko; Honda Research and Development, Japan

Shishin, Denis; University of Queensland, Australia

Shuleshova, Olga; Germany

Sierros, Konstantinos; West Virginia University, United States

Silva, Nila; SQM Industrial S.A., Chile

Simard, Alain; Pyrotek Incorporated, Canada

Sims, Zachary; Oak Ridge National Laboratory, United States

Sing, Arunima; National Institute of Standards and Technology, United States

Singh, Gurpreet; Kansas State University, United States
Singh, Gyanender; Oak Ridge National Laboratory, United States

Singh, Prabhakar; University of Connecticut Center for Clean Energy Engineering, United States

Skokov, Konstantin P.; Technische Universitat Darmstadt, Germany

Slaughter, Andrew; Idaho National Laboratory, United States

Slocik, Joseph M.; University Educational Services Incorporated, United States

So, Kang Pyo; Massachusetts Institute of Technology, United States

Sohn, Seok Su; Pohang University of Science Technology, South Korea

Sohn, Sungwoo; Yale University, United States

Somani, Vikas; Brazeway Incorporated, United States

Song, Jie; Colorado School of Mines, United States

Song, Miao; University of Michigan, United States

Sopu, Daniel; Germany

Souza, Eduardo; Villares Metals, Brazil

Stebner, Aaron; Colorado School of Mines, United States

Steinacker, Stephan; Montanuniversitaet Leoben, Austria

Steinbach, Ingo; Ruhr-University Bochum, Germany

Steinlechner, Stefan F.; Montanuniversitaet, Austria

Steube, Andreas; Hydro Aluminum Rolled Products GmbH, Germany

Steurer, Walter; ETH Zurich, Switzerland

Stoica, Mihai; Germany

Strutzenberg, Louise; NIST Marshall Space Flight Center, United States

Subramanian, Ramesh; Siemens Energy, United States

Sun, Haikuo; China ENFI Engineering Corporation, China

Tadokoro, Jun; Fueukawa Electric Company Ltd., Japan

Takemura, Masahiro; Japan Science and Technology Agency, Japan

Takizawa, Yoichi; Kyushu University, Japan
Tan, Krishda; Rio Tinto, Australia

Tang, Bin; Northwestern Polytechnical University, China

Tenorio, Jorge A. S.; University Sao Paulo, Brazil

Thomas, Derek; Made In Space, Incorporated, United States

Thomas, Vinoy; University of Alabama at Birmingham, United States

Thornton, Robert L.; Magnesium Elektron, United Kingdom

Tian, Mengkun; University of Tennessee, Knoxville, United States

Tian, Yanhong; Harbin Institute of Technology, China

Tian, Zhongliang; Cental South University, China

Timmel, Klaus; Helmholtz-Zentrum Dresden-Rossendorf, Germany

Todd, Madelein; Manganese Metal Company, South Africa

Toniolo, Pablo; Graftech International, United States

Tran, Thanh; Naval Sea Systems Command, United States

Tricker, David; Materion, United Kingdom

Troparevsky, Claudia; Oak Ridge National Laboratories, United States

Tsai, Che-Wei; National Tsing Hua University, Taiwan

Turgut, Zafer; Air Force Research Laboratories, United States

Unger, Alois; Aurubis Ag, Germany

Valloton, Jonas; University of Alberta, Canada

Van Den Berg, Willem; Graftech International, United States

Van Zee, John, W.; University of Alabama Tuscaloosa, United States

Vendette, Hugues; Bechtel, Canada

Verdenik, Anton; Talum Tovarna Aluminija, Slovenia

Vieregge, Klaus; Hydro Aluminum Rolled Products GmbH, Germany

Voelker, Bernhard; University of Leoben, Austria

Von Lilienfeld, Anatole; University of Basel, Switzerland 
Vora, Shailesh; Department of Energy/ National Energy Technology Laboratories, United States

Voter, Arthur F.; Los Alamos National Laboratories, United States

Voyer, Robert; Hatch, Canada

Vutova, Katia; Institute of Electronics, Bas, Bulgaria

Wada, Shohei; Kobe Steel, Japan

Waernes, Aud Nina; Sintef Materials and Chemistry, Norway

Walker, Peter; Otto Fuchs KG, Germany

Wall, James J.; Electric Power Research Institute, United States

Wang, Biao; Kunming University of Science and Technology, China

Wang, Cai-Zhuang; Ames Laboratories/ lowa State University, United States

Wang, Jingsong; University of Science and Technology, Beijing, China

Wang, Jun; Northwestern Polytechnical University, China

Wang, Xiaodong; Zhejiang University, China

Wang, Yun; BCAST-Brunel University, United Kingdom

Waz, Emmanuel; Constellium Technology Center, France

Weaver, Kevan; Terrapower LIc, United States

Wedde, Geir; Norwegian Emission Abatement Technologies, Norway

Weiler, Jon; Meridian Lightweight, Canada

Wenzel, Marco; TU Dresden, Germany

Whalen, Scott; Pacific Northwest National Laboratory, United States

Whiting, Justin G.; National Institute of Standards and Technology, United States

Whitsitt, Jacob; Alcoa, United States

Wicker, Ryan B.; University of Texas at El Paso, United States

Wilde, Gerhard H.; Uni Muenster, Germany

Williams, Edward J.; Quintus Technologies, United States
Winther, Grethe; Technical University of Denmark, Denmark

Wippermann, Andi; Leibniz Universitaet Hannover, Germany

Wojewoda-Budka, Joanna; Institute of Metal and Material Science, Poland

Wolf, Marcus; Federal Institute for Materials Research, Germany

Wolff, Martin; Helmholtz-Zentrum Geesthacht, Germany

Wondrak, Thomas; Helmholtz-Zentrum Dresden - Rossendorf, Germany

Wong-Ng, Winnie K.; National Institute of Standards and Technology, United States

Woo, Ki Suk; LG Electronics, South Korea

Woolum, Connor; Idaho National Laboratory, United States

Wu, Kunhua; Kunming University of Science and Technology, China

Xiao, Kai; Oak Ridge National Laboratory, United States

Xie, Guogiang; Tohoku University, Japan

$\mathrm{Xu}$, Ben; Tsinghua University, China

Xue, Liping; Flow Science Inc, United States

Yakubtsov, Igor; Integrity Testing Laboratory Inc, Canada

Yamada, Rui; Frontier Research Institute for Interdisciplinary Sciences, Japan

Yamamura, Yuji; Sumitomo Corporation, Japan

Yamashita, Ichiro; Nara Institute of Science and Technology, Japan

Yan, David P.; University of Wisconsin Green Bay, United States

Yanar, Cagatay; Alcoa, United States

Yang, Jianhua Joshua; University of Massachusetts Amherst, United States

Yang, Suyuan; Beijing Institute of Technology, China

Yang, Yong; General Electric, China

Yang, Zhigang; Tsinghua University, China

Yatsugi, Kenichi; Toyota Central R\&D Laboratories, Inc, Japan
Ye, Guozhu; Swerea Mefos, Sweden

Yi, Sangbong; Magic-Magnesium Innovation Centre, Germany

Yoon, Chang-Ho; Foosung Precision Ind Company, Ltd, South Korea

Yoshitake, Shunichi; Mitsubishi Materials Company, Japan

Youssef, Khaled M.; Qatar University, Qatar

Yu, Dong-Yurl; Kitech, South Korea

Yu, Min-Feng; Georgia Institute of Technology, United States

Zaloznik, Miha; CNRS, France

Zamora, Richard; Los Alamos National Laboratory, United States

Zeng, Li; Central South University, China

Zhang, Chi; Tsinghua University, China

Zhang, Duchao; Central South University, China

Zhang, Feng-Yuan; University of Tennessee Space, United States

Zhang, Xianguang; Tohoku University, Japan

Zherdev, Aleksey; Rusal ETC, Russian Federation

Zhou, Youdong; GE Global Research Center, United States

Zhu, Ruijun; China ENFI Engineering Corporation, China

Zhu, Tiejun; Zhejiang University,China

Zhu, Xueyu; University of Utah, United States

Zieba, Pawel; Polish Academy of Sciences, Poland

Zimmermann, James W.; Corning, United States

Zimmermann, Martina; TU Dresden, Germany

Zou, Xingli; Shanghai University, China

Zusheng, Hang; Nanjing Institute of Technology, China 